\title{
Presente y futuro de la radio digital: factores tecnológicos determinantes en su adopción y desarrollo
}

\author{
José María Huerta \\ Investigador independiente \\ Rebeca Amieva de la Vega \\ Universidad Complutense de Madrid \\ Adelaida Bolea de Anta \\ Universidad Complutense de Madrid
}

\section{Palabras clave}

Radio Digital, Estándar Radio DNS, Regulación, DAB, DRM, EURO-CHIP

\section{Resumen}

Este trabajo de investigación tiene como objetivo realizar un balance panorámico del mundo de la radio digital, a partir del análisis de diferentes estudios sobre la situación actual y perspectivas del sector radiofónico. Estos estudios han sido llevados a cabo por distintos organismos internacionales y nacionales, en algunos casos, de difusión exclusiva entre sus socios. Se han tenido en cuenta las aportaciones obtenidas del contacto directo con responsables y profesionales de primer nivel.

Entre nuestros objetivos, también está aclarar algunos conceptos clave (DAB, DRM, EURO-CHIP, DNS), necesarios para comprender la actualidad de la radio digital desde un punto de vista técnico. 


\title{
Present and future of digital radio: Technological key factors for its adop- tion and development
}

\section{Keywords}

Digital radio, DNS Standard Radio, Regulation, DAB, DRM, EURO-CHIP

\begin{abstract}
We have overviewed the Digital Radio world, from the analysis of different studies on the current situation and sector prospects. Such studies have been realised by several international and national organisations, in some cases, only shared between members. We have also considered other contributions from our direct contact with people in charge and top professionals. And finally, among our targets, we want to clarify some key terms (DAB, DRM, EURO-CHIP, DNS), needed to clarify what happens.
\end{abstract}

\begin{abstract}
Autores
José María Huerta [gjha@icam.es] es ingeniero y abogado. Ha sido director técnico de RNE y también miembro del Comité técnico de la European Broadcasting Union (EBU). En la actualidad es Director del Gabinete jurídico Huerta y Asociados, desde el que asesora a importantes empresas españolas en el ámbito de la comunicación.

Rebeca Amieva [amievadelavega@gmail.com] es licenciada en Comunicación Audiovisual por la UCM. Es Máster universitario en Cine, televisión y medios interactivos en la Universidad Rey Juan Carlos (URJC). En la actualidad es Técnico de Comunicación en el Departamento de Ventas, Marketing y Comunicación del Centro Universitario de Tecnología y Arte Digital, U-tad. Realiza su tesis doctoral en la UCM bajo la Dirección de la Dra. Adelaida Bolea de Anta.

Adelaida Bolea de Anta [adelaidabolea@ccinf.ucm.es ] es economista y doctora en Publicidad por la Universidad Complutense de Madrid (UCM). Profesora titular, es docente de estructura y empresa de radio de la citada Universidad. También imparte clases en el Master Radio de RNE-UCM.
\end{abstract}




\section{Introducción}

El 23 de julio de 1999 se aprobaba el Plan Técnico Nacional de Radiodifusión Sonora Digital Terrenal (Real Decreto 1287/1999), en el que se planificaba la digitalización de la tecnología terrestre para la difusión de la radio en España. El estándar elegido fue el DAB (Digital Audio Broadcasting) desarrollado por la EBU (European Broadcasting Union) y la Unión Europea dentro del proyecto Eureka 147, adoptado en 1995 por la ETSI. El DAB proporcionaba mejoras significativas en la gestión del espectro, mayor calidad del audio y la incorporación de servicios interactivos y de datos (Martí, Gutiérrez y Martínez, 2012: 2).

Sin embargo, tras la adjudicación de frecuencias nacionales y la planificación de los bloques de frecuencias locales (Orden del 15 de Octubre de 2001), el proceso de implantación del DAB quedaba paralizado, sucediéndose moratorias en su despliegue (Franquet, 2008: 21) y sin contenidos específicos que incentivasen la migración a la tecnología digital (Bolea, 2010: 2).

Doce años después del primer Plan Técnico, el 10 de junio de 2011 se aprobaba por el Real Decreto 802/2011, el nuevo Plan Técnico Nacional de la Radiodifusión Sonora Terrestre, reduciendo la cobertura al 20\% (Madrid y Barcelona) e instaurando el principio de neutralidad tecnológica, a la espera de las decisiones tomadas dentro del Foro de la Radio Digital.

El fracaso de la radio digital en España no es aislado y otros Estados se encuentran en una situación similar (WORLD DMB. Global Digital Radio Broadcasting Update. Septiembre 2012) Países como Dinamarca, Noruega, Suecia, Alemania y Reino Unido, tienen un gran desarrollo digital, en cobertura, estaciones de radio, contenidos diferenciados de los programas analógicos y regularidad en las emisiones, otros países como Australia, Bélgica, China, La república Checa, Ghana, Hong Kong, Malta, Holanda, Corea del sur y Suiza, tienen regulado el servicio y programación regular, aunque no esté completamente implantado.

Por otra parte, hay una serie de países, que sin tener emisiones regulares, sí que están haciendo ensayos e incluso en algunos casos han desarrollado una regulación, es el caso de España, pero también de otros países como Austria, Brunei Darussalam, China Taipei, Croacia, Francia, Hungría, Indonesia, Irlanda, Israel, Italia, Kuwait, Malaysia, Nueva Zelanda, Polonia, Sud Africa yVietnam.

Por último, cabe destacar a un cierto número de países, que simplemente han mostrado interés por la radio digital, entre ellos estarían Canadá, Estonia, Grecia, India, Lituania, Méjico, Mónaco, Namibia, Portugal, Rusia, Slovakia, Slovenia y Turkia.

Sin duda, el papel que ha jugado la Comisión Europea en el desarrollo de políticas ambiguas y poco claras, defendiendo la neutralidad tecnológica (Bonet et al, 2009: 87) ha impedido el desarrollo industrial del DAB y obstaculizado un mercado único de receptores. Además, nunca se planteó un mercado común para la radio digital en Europa, ni se introdujo dentro de su agenda digital. De 
esta manera, nos encontramos con que la legislación y puesta en marcha de la radio digital, ha conducido a la toma de decisiones soberanas de cada país, produciéndose estas, en diferentes intervalos de tiempo.

En las siguientes líneas nos sumergiremos en los aspectos técnicos de la radio digital, con el objetivo de dar a conocer los retos tecnológicos a los que se enfrenta la radio en un escenario claramente convergente.

\section{Metodología}

Los autores del artículo hemos partido de una revisión bibliográfica y un análisis de la legislación y los documentos oficiales de los distintos países de la UE. en relación a la radio digital.

A partir de ahí, se ha incorporado la información obtenida de estudios llevados a cabo por distintos organismos internacionales y nacionales, en algunos casos, de difusión exclusiva entre sus socios. También hemos tenido en cuenta las aportaciones obtenidas del contacto directo con responsables y profesionales de primer nivel.

\section{1. ¿De qué hablamos cuando nos referimos a la digitalización de la radio?}

La velocidad del cambio y las presiones comerciales que lo acompañan conducen a diferentes definiciones de radio digital, que según la plataforma o el soporte técnico puede significar diferentes cosas.

Para Ribes (2002:7), digitalización significa «el proceso por el cual una señal analógica se convierte en una señal digital. Dicho de otra forma, es representar mediante números la forma continua de una onda».

Existen diferentes estándares de transmisión digital terrestre para la difusión de los servicios radiofónicos. Normalmente, cuando hablamos de digitalización de la radio nos referimos a estos estándares y al abandono de la OM y la FM.

Sin embargo, existen definiciones más amplias del concepto digitalización que abarcan una comprensión más comercial. Estos puntos de vista consideran a la audiencia como un todo, independientemente de si la escucha de radio es lineal o mediante productos editorializados (podcast). Nos referimos por tanto, a otros tipos de emisión digital en Internet:

- Las estaciones de radio que tienen web.

- Presencia en otros portales web, como las redes sociales.

- Streaming de audio, a través de redes IP fijas/inalámbricas para ordenadores personales.

- Streaming a través de redes UMTS a los teléfonos móviles (3G). 
- Descarga de contenidos radiofónicos (podcast).

- Presencia en otros dispositivos conectados como videoconsolas (IP).

Estas diferentes interpretaciones pueden tener un efecto significativo sobre el coste de distribución multiplataforma de un servicio de radio, sin tener necesariamente una ventaja clara en términos de ingresos.

Los costes dependerán del número y tipología de las tecnologías de distribución que utilice cada emisor, de la calidad del servicio, del número de programas y en ocasiones, del número de oyentes (EBU, 2011a: 23). Las redes terrestres posibilitan economías de escala siendo los costes de distribución, constantes e independientes del número de oyentes. Sin embargo, en Internet los costes de distribución dependen del número de oyentes (cantidad de conexiones) y de la calidad del servicio. Mientras las tecnologías terrestres permiten a todos los ciudadanos el acceso gratuito e igualitario, también posibilitan un mayor control sobre la distribución de esos contenidos. Por el contrario, en Internet el control de esa difusión queda en manos de los ISP (Internet Service Providers), es decir, los operadores de telecomunicaciones.

Por todo lo expuesto anteriormente, entendemos que cuando hablamos de radio digital no solo podemos referirnos a los estándares de emisión sino que tenemos que contemplar otras formas de recepción digitales que nos introducen en un futuro híbrido.

\section{La ecuación Digital}

Los aspectos que influyen en el desarrollo de la radio digital son múltiples, la necesidad de crear contenidos diferenciados de los que emite la radio analógica, la comercialización de los espacios, la financiación de los equipos, etc. Nosotros nos centraremos en los aspectos técnicos y en este sentido, el anterior Director Adjunto de Tecnología y Desarrollo en la Unión Europea de Radio, David Wood (2012:11) trazó cinco cuestiones clave, que los radiodifusores deben de sopesar ante el desarrollo e implantación de la radio digital. Estos cinco principios atienden a diferentes cuestiones tecnológicas que nos parece imprescindible ampliar y analizar:

\subsection{Receptores de radio}

En primer lugar, los radiodifusores deben decidir cómo persuadir a los oyentes para que éstos compren receptores digitales. Como ya se ha mostrado en el caso español (Bolea, 2010:2), parece imprescindible que la radio digital deba ofrecer algo más que la radio analógica. Hablaríamos, por tanto, de calidad de audio y de contenidos, pero también del papel de la Administración en el impulso de la adopción de tecnologías digitales para la distribución de la radio y por supuesto, del decisivo papel de la industria tecnológica en la fabricación y desarrollo de 
receptores y prototipos, móviles y fijos, sin olvidar la posición de la industria automovilística.

Los problemas de la incompatibilidad de los receptores para los diferentes sistemas de recepción, digital y analógico, parece que ya han sido superados con el prometedor desarrollo del Euro-Chip (dispositivo híbrido). Este dispositivo posibilita la recepción analógica en FM y las señales digitales (DAB/DAB+/DMB) y puede ser fácilmente instalado en dispositivos móviles o coches. El Euro-Chip permite la recepción y sincronización de diferentes tipos de señales mediante diversas APP.

El reto del Euro-Chip consistiría en la adopción e introducción de este dispositivo en cualquier tipo de receptor, especialmente en los smartphones y tablets. De esta manera, los oyentes podrían disfrutar gratuitamente de las emisiones radiofónicas, independientemente de si son analógicas o digitales y lo que es más importante, el oyente podría acceder a servicios de valor añadido, mediante conexiones de banda ancha, que enriquecerían la escucha de los contenidos radiofónicos sin un sobre coste para el radiodifusor. Así, el Euro-Chip se postula como una de las opciones para atraer de nuevo a la audiencia joven, más interesada en este tipo de dispositivos inteligentes y cuyas posibilidades de negocio son más que prometedoras.

Sin embargo, parece indispensable que el desarrollo del Euro-Chip deba estar sujeto a licencias de código abierto, permitiendo la mejora de las herramientas y el perfeccionamiento de sus aplicaciones.

\subsection{Estándares técnicos de emisión digital terrestre}

La segunda cuestión clave es la elección de los estándares de emisión para la radio digital terrestre. En Europa encontramos las familias DAB (Digital Audio Broadcasting) y DRM (Digital Radio Mundiale), en USA el estándar elegido es el HD-Radio y el ISDB-Tn en Japón. Cada estándar tiene sus fortalezas y debilidades que no solo atienden a cuestiones tecnológicas.

La familia DAB trabaja en la banda VHF III y en la práctica ha dado lugar a la introducción de nuevos competidores o estaciones de radio, pues se readjudica la posición de cada estación en el dial por zonas de cobertura y por la posibilidad de desconexiones territoriales.

El estándar DRM se adopta para la digitalización de la Onda Media, mientras que el HD-Radio y el DRM+, podrían digitalizar la banda de FM sin necesidad de un nuevo concurso, para la asignación en el dial de los radiodifusores.

En el contexto europeo, a la vez que parece imprescindible la adopción combinada de DAB+ y DRM/DRM+ (EBU, 2011a:13) digitalizando todas las bandas de frecuencias en las que se distribuyen contenidos radiofónicos (OM y FM); llega lo que se conoce como radio híbrida. 
La Unión Europea de Radio argumenta los siguientes motivos para una difusión combinada entre redes terrestres y tecnología IP, mediante la utilización de tecnologías híbridas:

«Digital terrestrial broadcasting is the only way to deliver radio to mass audiences efficiently and cheaply, both in terms of distribution, and of consumption. At the same time, the internet offers unique opportunities for building communities and for attracting younger listeners. ” (EBU, 2011b: 2)

El estándar Radio DNS permite la recepción de FM, DAB, HD Radio, etc. junto a servicios ofrecidos mediante tecnología IP; a través de licencias de uso y explotación de código abierto ${ }^{1}$. Quizás el aspecto más interesante de esta tecnología sea el desarrollo de tres aplicaciones que introducen servicios de valor añadido a través de conexiones de Banda Ancha: RadioVIS, RadioEPG y RadioTAG ${ }^{2}$.

- RadioVIS: permite la recepción de imágenes y texto. Estos contenidos pueden enriquecer la emisión tradicional introduciendo información y publicidad.

- RadioEPG: proporciona información al oyente sobre la parrilla de programación de la emisora que está escuchando y las posibilidades de recepción de la misma. Así, el oyente puede combinar diferentes tecnologías y elegir servicios en streaming o mediante redes terrestres.

- RadioTAG: permite la interacción con el oyente. Al presionar un botón el oyente puede marcar un contenido como interesante y buscar más información en su teléfono móvil o en su ordenador. Esta aplicación permite la compra de entradas o música, ofrece información adicional y conecta a los oyentes con los anunciantes.

Por lo tanto, no se plantea que la difusión por Internet sustituya la difusión por redes terrestres, sino que la combinación de ambas tecnologías suponga unir las fortalezas de dos tipos de emisión, atendiendo tanto a la emisión masiva (generando economías de escala) como a la emisión de contenidos especializados en movilidad o de manera estacionaria.

\subsection{Calidad de audio}

La calidad de sonido que se obtiene con la tecnología DAB depende del ancho de banda que se asigne a cada programa, concepto que con la tecnología digital se asimila a la velocidad de transmisión, y esta a su vez depende del sistema de compresión: $\mathrm{DAB}$ o $\mathrm{DAB}+$, que se use para la comprensión de la señal de audio de cada programa.

La calidad de los programas en DAB cuando se utiliza un ancho de banda elevado, por ejemplo una velocidad de transmisión de 256 kilobit por segundo (kbit/s), es similar a la del disco compacto (CD), pero no es exactamente la misma aunque

1 http://radiodns.org/for/manufacturers/ (Consultado el 28/12/12)

2 http://radiodns.org/ (Consultado el 28/12/12) 
al oído suene aparentemente igual, ya que para poder emitir en la radio digital varios programas por el mismo transmisor es necesario reducir la información de la señal de audio de cada programa, eliminando la información que el oído no es capaz de percibir. A este proceso de reducción de la información se le denomina compresión de la señal.

Es posible transmitir programas en DAB con ancho de banda más reducidos pero la calidad que se obtiene está siempre en función del ancho de banda y la protección que se le quiera asignar frente a los errores que se producen, en la transmisión, propagación y recepción de la señal, hasta que llega al receptor.

Por ejemplo, podríamos transmitir 6 programas con calidad $192 \mathrm{kbit} / \mathrm{s}$ por el mismo transmisor, lo que se conoce con el nombre de múltiple, con un grado de protección 3 para el audio de cada programa, dejando una capacidad restante de $32 \mathrm{kbit} / \mathrm{s}$ para el canal de datos de cada programa, dado que la capacidad útil del múltiple, es decir su ancho de banda útil total es de aproximadamente1,5 Megabit por segundo (Mbit/s).

Sin embargo, si transmitiéramos con una calidad inferior por ejemplo 160 $\mathrm{kbit} / \mathrm{s}$, con el mismo grado de protección 3, podríamos transmitir 7 programas por el mismo múltiple. En el caso de que se optara por la misma calidad de 160 kbit/s pero con una protección superior, de grado 2, en lugar de 7 programas solamente podríamos transmitir 5 programas por múltiple.

La calidad de los programas en DAB puede estructurarse y configurarse dinámicamente, permitiendo acomodar calidades de transmisión entre 8 y $380 \mathrm{kbit} / \mathrm{s}$ incluyendo la protección adecuada.

El tercer paso es decidir qué calidad de audio se debe suministrar para los servicios de radio digital. Esto puede ser una solución de compromiso en términos de contenido y tiempo de audiencia. Por un lado, cuanto menor sea el ancho de banda que se utilice por estación, mayor será el número de las estaciones que se podrá meter en un mismo ancho de banda total. El bajar el ancho de banda por estación puede significar mayores posibilidades de elección para el oyente, mayores ingresos para los Estados por los derechos de licencias de las estaciones concedidas y tal vez mayores ingresos publicitarios.

\subsection{Sistemas de compresión de la señal}

El sistema de compresión de la señal de audio de cada programa que se utilizaba con la tecnología DAB se denominó Musicam, mas tarde se normalizó con el nombre de MEPG2 o MP2. Es un sistema de compresión similar al MP3 pero que necesita menor capacidad de procesamiento que este.

Se basa fundamentalmente, como hemos dicho antes, en eliminar la información que el oído no puede distinguir aprovechando el efecto de enmascaramiento que se produce en el oído humano debido a sus características psicoacústicas, ya 
que no es capaz de percibir todos los sonidos presentes en un momento dado, y por tanto no es necesario transmitir las señales que no son audibles.

Cuando hay dos sonidos muy próximos en frecuencia y uno de ellos es más fuerte que el otro resulta que el sonido que tiene el nivel inferior queda enmascarado y no es posible oírlo, por tanto no es necesario transmitirlo. Además el oído tiene un umbral de ruido por debajo del cual no oye los sonidos y por tanto tampoco es necesario transmitir los sonidos que se encuentran por debajo de dicho nivel de ruido. De esta forma se consigue reducir la información original, y por tanto el ancho de banda original, que se necesita para transmitir cada programa. Por ejemplo, en el caso de que comprimiéramos la información original de un programa en un factor de 6 veces, sería posible transmitir 6 programas comprimidos, utilizando el ancho de banda original necesario para transmitir un único programa.

El sistema DAB nació en 1987 dentro de un proyecto europeo conocido como Eureka 147. Es un sistema que está utilizando una tecnología que hoy día no es la óptima. La evolución de la tecnología de procesamiento de señales digitales ha permitido diseñar sistemas de compresión más eficientes.

Para mejorar la eficiencia en la compresión de audio del DAB el WorldDMB fórum desarrolló un nuevo sistema de compresión denominado $\mathrm{DAB}+$ que es dos veces más eficiente que el utilizado en el $\mathrm{DAB}$. El DAB+ utiliza la compresión denominada HE-AACv2 más conocida como MP4, que permite transmitir el mismo programa aún con menor ancho de banda.

Por otro lado, utilizar un sistema de compresión más moderno supone reducir el ancho de banda necesario, para evitar recibir deficiencias motivadas por la compresión, cuando se emite un material de audio de tipo medio, por ejemplo un programa de radio de tipo general, pero la reducción de ancho de banda no es tanta cuando se utiliza material de audio de tipo crítico, como por ejemplo música clásica. Además, el aumento del número de estaciones de radio en un determinado mercado puede tener un lado negativo para la «calidad de contenidos», ya que podría ser necesario producir más programación sin un aumento en el presupuesto general o en el tamaño del mercado.

\subsection{Otros servicios de audio competidores y tiempo de escucha}

Por último, debemos referirnos a la relación entre tiempo de escucha y calidad del audio. Lo más sensato sería pensar que a mayor calidad de audio el oyente tendería a escuchar por más tiempo. Sin embargo, algunos estudios demuestran (Gutiérrez, Ribes y Monclús, 2012: 18) que los hábitos de escucha de los oyentes no solo se basan en estos parámetros y que son los contenidos los que retienen al oyente. Plataformas de éxito como Spotify o Youtube, alojan contenidos cuya calidad de audio está muy por debajo de la calidad CD. Habituados a la escucha en formato MP3, la calidad de audio para ciertos contenidos no parece determinante. 
No obstante, debemos referirnos aquí, a las posibilidades comunicativas que ofrece el sonido envolvente 5.1 para la producción y difusión de conciertos en alta calidad $\mathrm{u}$ otros eventos en los que la calidad del sonido pueda ser un aliciente para su escucha (Sánchez et al., 2009).

\section{Conclusiones}

Aunque la mayoría de los países de la UE hayan legislado la política de digitalización de la radio, sólo unos pocos han realizado una implantación, con un grado de penetración en la población y una cobertura del país, que pudiéramos calificar de éxito.

Una buena parte del fracaso, se le debe adjudicar a la Comisión Europea (CE), que ha mantenido una política ambigua al respecto, lo que ha hecho imposible el desarrollo industrial del DAB.

Paralelamente, el desarrollo tecnológico ha dejado obsoleta la tecnología DAB y los países que se lanzaron en un primer momento a la digitalización se encuentran ahora ante un serio problema: si quieren cambiar a la tecnología DAB+ es necesario cambiar los equipos receptores y eso políticamente es difícil de comunicar a los oyentes.

La posición de los fabricantes, también debe ser tenida en cuenta ya que en su caso, ante la incertidumbre sobre la tecnología a decidir para el conjunto de la UE, no están dispuestos a correr riesgos, invirtiendo en nuevos modelos de receptores, de dudosa venta.

En este sentido, el Euro-Chip (dispositivo híbrido) vendría a resolver los problemas de incompatibilidad entre receptores, ya que posibilita la recepción analógica en FM y las señales digitales (DAB/DAB+, DMB) y se podría insertar en cualquier dispositivo de móviles o coches, especialmente en smartphones y tablets, el reto sería que se pudiera insertar en cualquier tipo de receptor.

Por otra parte, el estándar de radio DNS permite la recepción de FM, DAB, HD Radio, etc. Junto a servicios ofrecidos mediante la tecnología IP, además tiene tres aplicaciones que introducen servicios de valor añadido, a través de conexiones de Banda Ancha.

Lo mas razonable sería entender la digitalización en sentido amplio, abarcando una comprensión mas comercial, nos referimos a la emisión digital en Internet.

De una u otra forma, los concesionarios del servicio deberán evaluar el coste de distribución multiplataforma y las ventajas que cada una les reporta.

La posibilidad que ofrece la tecnología $\mathrm{DAB}+$, a los concesionarios del servicio, de ofrecer varios programas de forma simultánea, les permite generar contenidos muy especializados y eso atraería nuevos anunciantes para los que hasta 
ahora no resultaba interesante la publicidad en las emisoras de radio generalistas, con lo que se encontrarían con nuevas fuentes de financiación.

Dada la situación de incertidumbre que vive el proceso de digitalización en toda Europa, plantear una fecha de apagón analógico supondría una insensatez, pero avanzar en el despliegue de $\mathrm{DAB}+\mathrm{y} \mathrm{DRM} / \mathrm{DRM}+$ se torna cada vez más necesario.

Parece indispensable el establecimiento de acuerdos europeos, reevaluando las posibilidades de las diferentes tecnologías mencionadas y contemplando las características del espectro radioeléctrico en cada uno de los estados miembros.

En definitiva, consideramos necesario un plan europeo sobre la digitalización de la radio, que garantice la coordinación en su despliegue, fechas de apagón y un mercado común de receptores.

\section{Fuentes documentales}

Ala-Fossi, M.; Lax, S.; O’Neill, B.; Jauert, P. y H. Shaw (2008). The Future of Radio is Still Digital-But Which One? Expert Perspectives and Future Scenarios for Radio Media in 2015. En: Journal of Radio \& Audio Media, Vol. 15, $\mathrm{n}^{\mathrm{o}}$ 1, 4-25.

Arboledas, Luis (2010). La radio española: un negocio analógico en la era digital. En: Revista de Economía Política de las Tecnologías de la Información y Comunicación, Vol. XII, $\mathrm{n}^{0} 2$.

Bolea de Anta, Adelaida (2010): La radio que viene: ¿Hay alguien ahí? II Congreso Internacional AEIC. Málaga.

Bonet, Montse; Corominas, Maria; Fernández, Isabel; Mercè (2009). Keys to the Failure to DAB in Spain. En: Journal of Radio \& Audio Media, Vol.16, n ${ }^{0} 1$, 83-101.

EBU-UER (2011a). Technical Report 013: The Future of Terrestrial Broadcasting (Documento interno).

EBU-UER (2011b). Digital Radio in 2012 (Documento Interno)

Gallego Pérez, Ignacio (2012). La audiencia en la radio: viejos roles, nuevas funciones. En: Gallego Pérez, Ignacio y García Leiva, María Trinidad (coord.). Sintonizando el futuro: Radio y producción sonora en el siglo XXI. Madrid: Instituto RTVE.

Gutiérrez, María; Ribes, Francesc Xavier; Monclús, Belén (2012): Percepción de los jóvenes ante las estrategias programáticas de la radio musical de antena convencional y online. El caso de Cataluña. Comunicación presentada en el III Congreso Internacional AEIC. Tarragona. 
Ribes, Francesc Xavier (2001). Las emisoras de radio del Estado español en internet: las bitcasters. Tesis doctoral.

Sánchez Cid, Manuel; Benítez Iglesias, Antonio Jesús y García García, Francisco (2009). Estado de implantación del sonido envolvente 5.1 en el medio Radio en España. En: Icono 14, ${ }^{\circ} 13,239-258$.

Wood, David (2012). Solving the Digital Radio Equation. En: EBU Tech-i, $\mathrm{n}^{0} 12$, P. 11. Disponible en: http://tech.ebu.ch/docs/tech-i/ebu_tech-i_012.pdf. (01/02/13).

WORLD DMB (2012). Global Digital Radio Broadcasting Update.

\section{Otras fuentes documentales consultadas}

EBU (2013). Case Studies on the Implementation of DRM+ in Band II. Disponible en: http://tech.ebu.ch/docs/tech/tech3357.pdf (01/02/13).

EBU (2013). Digital Radio distribution in Europe. Disponible en: http://tech. ebu.ch/docs/r/r138.pdf (01/02/13).

EMARKETER (2011). Spain Digital Trends.

Parnell, Karen; Nordmann, Jan; Ogilvie, Alan (2011). IMDA making sense of Internet Radio. EBU Technical Review, Geneva, Switzerland. Disponible en: http://tech.ebu.ch/docs/techreview/trev_2011-Q3_imda_parnell.pdf (01/02/13).

\section{Referencia de este artículo}

Huerta, José María, Amieva de la Vega, Rebeca y Bolea de Anta, Adelaida (2013). Presente y futuro de la radio digital: factores tecnológicos determinantes en su adopción y desarrollo. En: adComunica. Revista Científica de Estrategias, Tendencias e Innovación en Comunicación, $\mathrm{n}^{\circ} 5$. Castellón: Asociación para el Desarrollo de la Comunicación adComunica, Universidad Complutense de Madrid y Universitat Jaume I, 55-66. DOI: http://dx.doi.org/10.6035/2174-0992.2013.5.5 